\title{
Long-term variability of the SX Phoenicis star CY Aquarii ${ }^{\star}$
}

\author{
J. N. Fu and C. Sterken ${ }^{\star \star}$
}

\begin{abstract}
Astronomy Group, Vrije Universiteit Brussels (VUB), Pleinlaan 2, 1050 Brussels, Belgium e-mail: csterken@vub.ac.be
\end{abstract}

Received 4 April 2003 / Accepted 18 April 2003

\begin{abstract}
We present 23 new times of maximum light of the SX Phoenicis star CY Aquarii. The O-C diagram reveals a new value of the period $P=0.061038394 \pm 0.000000006$ since 1996. CY Aqr is the first SX Phe star where the major component of the long-term period change can be represented without invoking large-amplitude period jumps. A linear period change combined with the light-time effect in a highly-eccentric orbit yields a reasonably precise solution. The binary explanation, however, does not account for the full magnitude of the period changes, and small residuals in the time interval 1930-1950 seem to remain.
\end{abstract}

Key words. stars: individual: CY Aqr - stars: SX Phe - stars: oscillations

\section{Introduction}

CY Aquarii (BD $\left.+0^{\circ} 4900\right)$ is a short-period $(87.9 \mathrm{~min})$ largeamplitude (0.71 mag in $V$ and $0.89 \mathrm{mag}$ in $B$ ) SX Phoenicis star. Since the discovery of its light variability in $1934, \mathrm{CY}$ Aqr has been extensively observed for almost seven decades. As the latest complete study on the long-term period change of CY Aqr, Powell et al. (1995, hereinafter PJM) listed 472 individual times of maximum since the original epoch of maximum light, including 88 new maximum times obtained over a time interval of 20 years. After summarising four different models for explaining the discrepancies between the observed and calculated times of maximum light, PJM concluded that the model of straight-line segments was to be preferred over the parabolic model, the binary hypothesis, and the explanation with discontinuous phase jumps. In addition, PJM alluded to another change of period to be expected some time in the coming years. Fu et al. (1994), Zhou et al. (1999) and Fu (2000), respectively, provided 10, 4 and 1 new times of maximum light. In the present paper, we give the results of new observations obtained since September 1999. In addition, we collected in Table 1 times of maximum which were not in the list of PJM.

\section{New observations}

CY Aqr was observed at Sierra Nevada Observatory (SNO, Spain) on September 10, 1999, with a 0.9-m telescope during a test of a CCD camera. The Johnson $V$ filter was used. At SaintVéran Observatory (SVO, France), a 0.62-m reflector equipped

Send offprint requests to: J. N. Fu, e-mail: jianfu@vub. ac . be

* Based in part on observations obtained at ESO La Silla, application 269.D-5038.

$\star \star$ Research Director, Belgian Fund for Scientific Research (FWO).

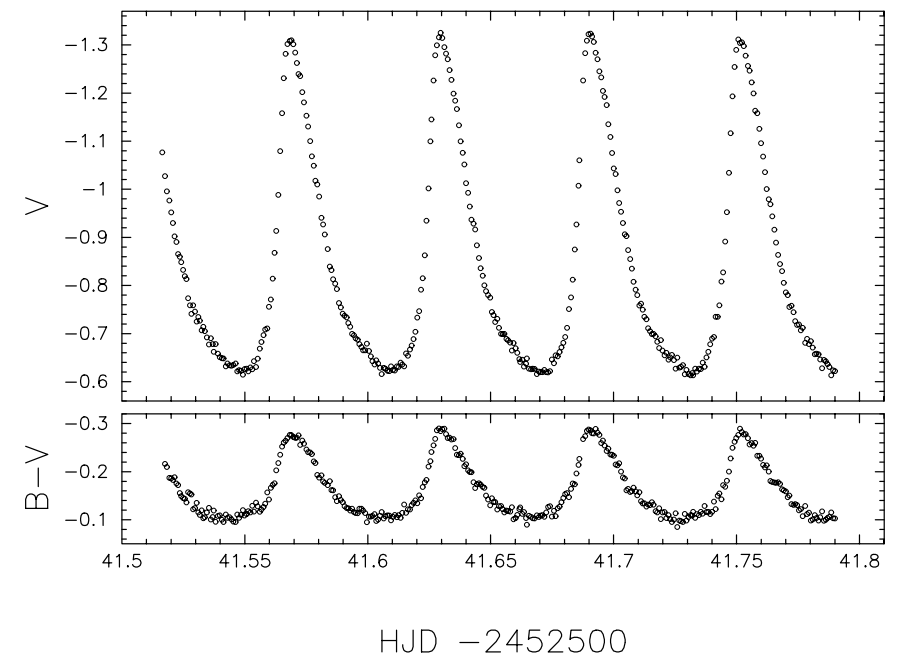

Fig. 1. Differential light curves $(V)$ and color curves $(B-V)$ for one single night.

with a ST-7 CCD camera was used from October 9 to 12, 1999 in white light. At ESO La Silla Observatory (Chile), we used the Danish 1.54-m telescope with DFOSC in Johnson $V$ and $B$ bands in September and December 2002. For all these observations, the constant star GSC 00567-01826 $(V=11.57)$ was used as comparison. Figure 1 shows the light curves in $V$ and the $B-V$ color curves of one night obtained at La Silla.

Table 2 lists the 23 times of maximum light determined from all our observations, 1 time calculated from the Hipparcos data (Perryman et al. 1997), and 2 times obtained by Jiang (2003) at Xinglong station (China). From the ESO $B$ time series, 16 times of maximum light were derived. The average difference between a maximum time in $B$ and $V$ amounts to 
Table 1. The maxima of CY Aqr in the literature but not in Table 1 of PJM. $T_{\max }$ is in HJD-2 400 000. Band: visual, photographic or Johnson $V$. Source: Ash = Ashbrook (1953), FJL = Fu et al. (1994), Fu = Fu (2000), GA = Gossner \& Ashbrook (1946), ZFJ = Zhou et al. (1999).

\begin{tabular}{|c|c|c|c|c|c|c|c|c|c|c|c|}
\hline $\bar{T} T_{\max }$ & Band & Source & $\overline{\bar{T}} \overline{\max }$ & Band & Source & 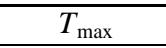 & Band & Source & $\bar{T} \overline{T_{\max }}$ & Band & Source \\
\hline 29079.8141 & vis & GA & 31751.4716 & vis & GA & 33171.7111 & vis & Ash & 49281.1390 & $V$ & FJL \\
\hline 29085.7353 & vis & GA & 31751.5291 & vis & GA & 33171.7737 & vis & Ash & 49282.0540 & V & FJL \\
\hline 29085.7950 & vis & GA & 31751.5302 & vis & GA & 33172.7509 & vis & Ash & 49282.1150 & $V$ & FJL \\
\hline 29085.8545 & vis & GA & 31758.3671 & vis & Ash & 33179.5285 & vis & Ash & 49282.1760 & $V$ & FJL \\
\hline 29086.7710 & vis & GA & 31759.5265 & vis & Ash & 33180.3789 & vis & Ash & 49283.0313 & $V$ & FJL \\
\hline 29086.8314 & vis & GA & 31765.5099 & vis & GA & 33180.6860 & vis & Ash & 49283.0915 & $V$ & FJL \\
\hline 29088.7853 & vis & GA & 31765.5107 & vis & GA & 33183.5557 & vis & Ash & 49328.9938 & V & FJL \\
\hline 29107.7670 & vis & GA & 31765.5694 & vis & GA & 33183.6161 & vis & Ash & 50072.9916 & $V$ & $\mathrm{Fu}$ \\
\hline 29108.7415 & vis & GA & 31765.5696 & vis & GA & 33185.5702 & vis & Ash & 50401.0125 & $V$ & ZFJ \\
\hline 31291.6650 & pg & Ash & 32092.3061 & pg & Ash & 45972.1828 & V & FJL & 50401.0735 & $V$ & ZFJ \\
\hline 31742.4357 & vis & Ash & 32445.7198 & vis & Ash & 49280.0396 & V & FJL & 50401.1345 & V & ZFJ \\
\hline 31750.4951 & vis & GA & 33099.8081 & vis & Ash & 49281.0776 & V & FJL & 50401.9888 & V & ZFJ \\
\hline 31751.4676 & vis & GA & 33171.6528 & vis & Ash & & & & & & \\
\hline
\end{tabular}

Table 2. New maxima of CY Aqr. Symbols as in Table 1. $w=$ "white" light. Obs: Observatory (XL = Xinglong station, Hip = Hipparcos satellite, SVO = St. Véran, ESO = La Silla).

\begin{tabular}{cccccccccccc}
\hline \hline$T_{\max }$ & Band & Obs & $T_{\max }$ & Band & Obs & $T_{\max }$ & Band & Obs & $T_{\max }$ & Band & Obs \\
\hline 45592.7682 & $V$ & XL & 51463.4468 & $w$ & SVO & 52539.6152 & $V$ & ESO & 52541.5683 & $V$ & ESO \\
45904.2456 & $V$ & XL & 51463.5085 & $w$ & SVO & 52539.6753 & $V$ & ESO & 52541.6293 & $V$ & ESO \\
48022.0354 & $V$ & Hip & 52534.6710 & $V$ & ESO & 52539.7366 & $V$ & ESO & 52541.6900 & $V$ & ESO \\
51432.5627 & $V$ & SNO & 52535.7086 & $V$ & ESO & 52540.6521 & $V$ & ESO & 52541.7514 & $V$ & ESO \\
51461.4940 & $w$ & SVO & 52538.6990 & $V$ & ESO & 52540.7134 & $V$ & ESO & 52625.5569 & $V$ & ESO \\
51462.4095 & $w$ & SVO & 52538.7600 & $V$ & ESO & 52540.7752 & $V$ & ESO & 52626.5339 & $V$ & ESO \\
51463.3864 & $w$ & SVO & 52539.5534 & $V$ & ESO & & & & & & \\
\hline
\end{tabular}

$-0.00005 \pm 0.00014$, and the standard deviation on a single time of maximum determined from the ESO observations is 0.0005 .

A frequency analysis of ESO $V$ and $B$ data was carried out using PERIOD98 (Sperl 1998). One single dominant peak at frequency $f=16.3822 \pm 0.0002 \mathrm{~d}^{-1}$ was found.

\section{Analysis of the $\mathrm{O}-\mathrm{C}$ diagram}

The O-C values of 548 times of 544 maxima (Table 1 of PJM plus Tables 1 and 2 of the present paper) were calculated using the ephemeris of Mahdy \& Szeidl (1980): HJD2426159.485 $+0.061038328 E$, where $E$ is the cycle number since HJD2426159.485. Figure 2 shows the resulting $\mathrm{O}-\mathrm{C}$ diagram. We made piece-wise linear fits in a similar way as PJM, and obtained the five straight-line segments shown in Fig. 2.

The straight-line fit to all the available times of maximum light yields the ephemeris formula

$C=\mathrm{HJD} 2426159.4967+0.0610383716 E$,

by which the resulting $\mathrm{O}-\mathrm{C}$ diagram displays a strong cyclic variation. The residuals from a sine function fit show a monotonous trend which can best be represented by a secondorder polynomial (higher-order polynomials do not yield significantly better fits).

Finally, a quadratic plus a function of sines of the form

$C^{\prime}=\mathrm{HJD}_{0}+P_{\mathrm{pul}} E+\frac{1}{2} \beta E^{2}+A \sin \phi+B \cos \phi$,

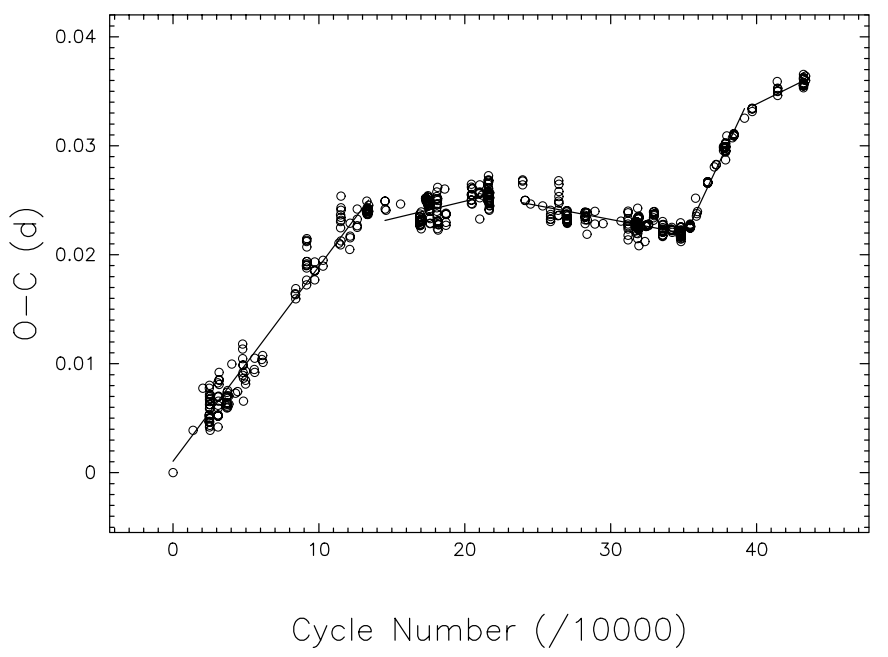

Fig. 2. O-C diagram of CY Aqr for all available times of maximum light. Straight-line fits to 5 segments is shown.

where $\phi$ is the solution of Kepler's equation

$\phi-e \sin \phi=\frac{2 \pi}{P_{\text {orb }}}\left(P_{\text {pul }} E-t_{0}\right)$

is fitted to the O-C points. Equation (2) is equivalent to Eq. (2) of Irwin (1952) and Eq. (1) of Ribas et al. (2002) and describes the light time effect (where $P_{\text {orb }}$ is the orbital period; $P_{\text {pul }}$ the pulsation period of the variable star; $t_{0}$ the time of periastron passage). 


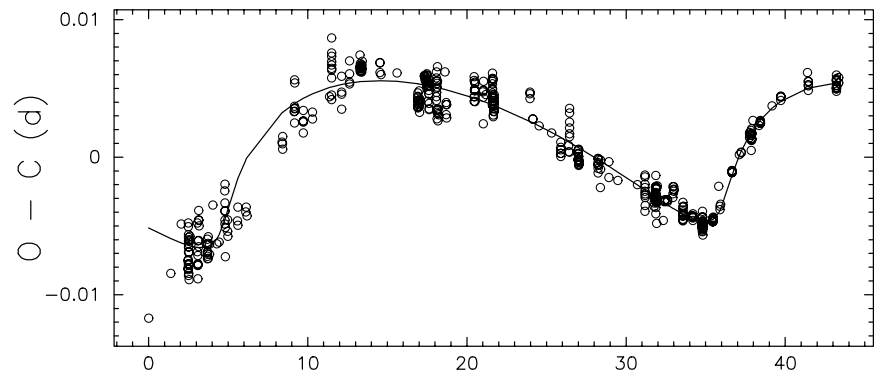

Cycle Number (/10000)

Fig. 3. O-C diagram of CY Aqr based on Eq. (1), the line is the fitted light-time solution.

Table 3. The 5 straight-line fits to the $\mathrm{O}-\mathrm{C}$ diagram.

\begin{tabular}{crrr}
\hline \hline Years & $\begin{array}{r}\text { HJD } \\
(-2400000)\end{array}$ & $\begin{array}{c}\text { Period } \\
(\text { days })\end{array}$ & $\begin{array}{c}P_{\text {frac }} \\
\left(10^{-6}\right)\end{array}$ \\
\hline $1930-1952$ & 26159.4861 & 0.061038507 & -2.3 \\
\pm 2 & \pm 3 & \\
$1953-1966$ & 35032.4057 & 0.061038360 & -0.9 \\
& \pm 2 & \pm 4 & \\
$1970-1988$ & 40779.7763 & 0.061038303 & 5.1 \\
& \pm 1 & \pm 2 & \\
$1989-1995$ & 47792.7730 & 0.061038614 & -3.6 \\
& \pm 1 & \pm 5 & \\
$1996-2002$ & 50401.0127 & 0.061038394 & \\
& \pm 2 & \pm 6 & \\
\hline
\end{tabular}

\section{Discussion}

The parameters of the 5 straight-line segments to the $\mathrm{O}-\mathrm{C}$ points in Fig. 2 are given in Table 3, which also lists the fractional period changes $P_{\text {frac }}=\left(P_{\text {final }}-P_{\text {initial }}\right) / P_{\text {initial }}$ for $1952,1966,1988,1995$. The first three values are comparable to those of Coates et al. (1994) and PJM. As is very well known, no models exist that can account for such abrupt period changes.

The sinusoidal oscillation is either caused by the light-time effect in a binary system, or by the beating effect of two pulsation modes which have very close frequencies. For the modebeating model, the fitting of $\mathrm{O}-\mathrm{C}$ points in Fig. 3 leads to a frequency separation of $4.6 \times 10^{-5} \mathrm{~d}^{-1}$.

In order to solve the light-time curve for orbital parameters, one needs to assess the uncertainties of the individual times of maximum. As discussed above, the standard deviation of a single time of maximum derived from our CCD data amounts to about $45 \mathrm{~s}$, however time series with lower temporal resolution and with less efficient detectors will yield deviations of several minutes at least. PJM, for example, give a relative (but arbitrary) weight for the data they list. We first proceeded by giving all photoelectric data the same weight as our own data, whereas photographic data were given a five times smaller weight. The solution obtained with the computer code of Ribas et al. (2002) results in a semiamplitude of $0.007, e=$ $0.77 \pm 0.01, P_{\text {orb }}=52.5 \pm 0.3 \mathrm{yr}$ and $\beta=(-0.64 \pm 0.10) \times 10^{-13} \mathrm{~d}$. Remaining orbital parameters are embedded in the coefficients $A$ and $B$, and it makes no sense to attempt a definite solution

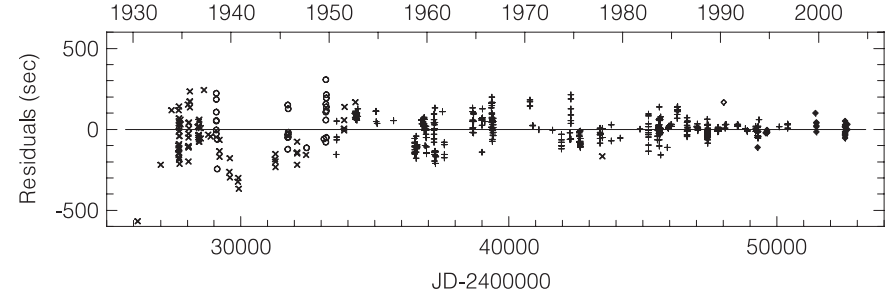

Fig. 4. Residual O-C diagram after subtraction of expression (2). $\times$ are photographic data, $\circ$ are visual, + stand for photoelectric, $\diamond$ is from Hipparcos data and filled diamonds are from CCD- $V$ data.

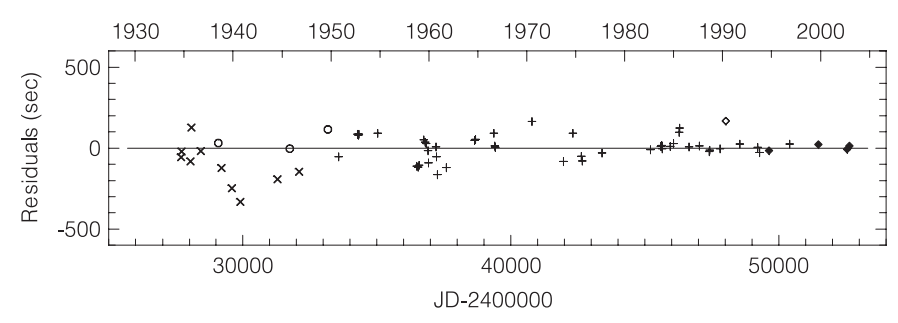

Fig. 5. Average O-C residuals, symbols as in Fig. 4.

since our data span only one cycle. This result, though seemingly precise, should not be considered as an accurate solution of the orbital parameters, because the impact of the arbitrarily chosen weights of the times of maximum is quite large. The extreme case with all weights equal, for example, leads to a solution with $e=0.70 \pm 0.01, P_{\text {orb }}=51.9 \pm 0.1 \mathrm{yr}$ and $\beta=(-0.86 \pm 0.04) \times 10^{-13} \mathrm{~d}$.

The quadratic term leads to the rate of period change of

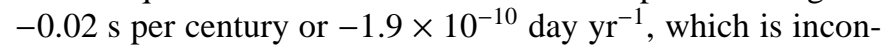
sistent with the evolutionary change calculated for early-postmain-sequence models (see, e.g., Breger \& Pamyatnykh 1998) - though we insist to respect Breger \& Pamyatnykh's warning that interpreting observed period changes as stellar evolution rates is appropriate only when dealing with a group of stars in the same stage of evolution (hence their conclusion that observed period changes in $\delta$ Scuti stars are caused by nonlinear pulsation effects rather than by an evolutionary mechanism).

Figure 4 shows the remaining residuals of the solution with separate symbols for the different type of detector. Evidently, the scatter around the zero line systematically decreases from photographic to CCD detectors. This is not surprising because not only are the photoelectric detectors more accurate (compared to the non-linear response of the visual system and the photographic plate), they also have a much higher quantum efficiency which renders the exposure times much shorter and hence produce more accurate light curves. Indeed, long exposure times (up to $30 \mathrm{~min}$ as mentioned by Hoffmeister 1934) result in much flatter light curves with times of maximum that are systematically earlier or later.

In order to verify whether all discrepancies can be explained by the detector, we have plotted in Fig. 5 average residuals derived from groups of 5 to 10 times of maximum belonging to a same detector and a time interval of less than 20 days. Symbols have the same meaning as in Fig. 4, error bars do not exceed the size of the symbol. It is evident that both the photographic and visual results obtained between 1935 and 1950 
display a same trend, which leads to the conclusion that the visual data are useful and should not be discarded (as was always done in the past), and also that the discrepancies cannot be assigned to the detector only. Indeed, the visual-data residuals display a smaller range than the photographic data, and the last photographic residuals are perfectly compatible with the subsequent photoelectric data.

\section{Conclusions}

The new data lead to an updated value of the pulsation period of CY Aqr of 0 $061038394 \pm 0.000000006$ since 1996. The representation of the $\mathrm{O}-\mathrm{C}$ diagram by a sequence of linear segments or by a combination of a continuous period change and the light time effect in a binary configuration seem equally acceptable. CY Aqr is at least one SX Phe star where the major component of the period variation can be represented without invoking large-amplitude abrupt period jumps. A linear period change combined with the light-time effect in a highly-eccentric orbit yields a reasonably precise solution, the accuracy of which strongly depends on the assigned weights of the photographic and visual data. The binary explanation, however, does not account for the full magnitude of the period change, and small residuals in the time interval 1930-1950 seem to remain.

Acknowledgements. This work has been supported by "IAP P5/36" Interuniversity Attraction Poles Programme of the Belgian Federal Office for Scientific, Technical and Cultural Affairs, the Belgian Fund for Scientific Research (FWO), the Flemish Ministry for Foreign
Policy, European Affairs, Science and Technology, under contract BIL 99/2 and by the Danish Natural Science Research Council through the centre for Ground-Based Observational Astronomy. JNF acknowledges financial support from Laboratoire d'Astrophysique of Toulouse Observatory (France) for the observing missions at SNO and SVO, the local support of Dr. R. Garrido at SNO and observing assistance by Mr. O. Thizy at SVO. The authors thank Prof. S.Y. Jiang for providing 2 unpublished times of maximum, and Dr. Ignasi Ribas for providing the light-time computing code.

\section{References}

Ashbrook, J. 1953, AJ, 59, 6

Breger, M., \& Pamyatnykh, A. A. 1998, A\&A, 332, 958

Coates, D. W., Fernley, J. A., Sekiguchi, K., et al. 1994, MNRAS, 266, 1

Fu, J. N., Jiang, S. Y., \& Liu, Y. Y. 1994, IBVS, 3970

Fu, J. N. 2000, ASP Conf. Ser., 203, 475

Gossner, J., \& Ashbrook, J. 1946, AJ, 52, 55

Hoffmeister, C. 1934, AN, 16, 45

Irwin, J. B. 1952, ApJ, 116, 211

Jiang, S. Y. 2003, private communication

Mahdy, H. A., \& Szeidl, B. 1980, Mitteilungen der Sternwarte Budapest, 74

Perryman, M. A. C., Lindegren L., Kovalevsky, J., et al. 1997, A\&A, 323, L49

Powell, J. M., Joner, M. D., \& McNamara, D. H. 1995, PASP, 107, 225

Ribas, I., Arenou, F., \& Guinan, E. F. 2002, ApJ, 123, 2033

Sperl, M. 1998, Comm. in Asteroseismol., 111, 1

Zhou, A. Y., Fu, J. N., \& Jiang, S. Y. 1999, Ap\&SS, 268, 397 